\title{
Designate a Healthcare Setting Representative
}

National Cancer Institute

\section{Source}

National Cancer Institute. Designate a Healthcare Setting Representative. NCI

Thesaurus. Code C128495.

The healthcare setting must designate an authorized representative to act on that setting's behalf. 